Dom. Cien., ISSN: 2477-8818

Vol. 4, núm. 1, enero, 2018, pp. 141-154

Sistemas de información empresarial: la información como recurso estratégico

\title{
Sistemas de información empresarial: la información como recurso estratégico
}

\section{Business information systems: information as a strategic resource}

\section{Sistemas de informação empresarial: a informação como um recurso estratégico}

José R. Moreno-Cevallos I

jr66me@hotmail.com

Beatriz L. Dueñas-Holguín II

beatriz.duena1@gmail.com

Recibido: 28 de septiembre de 2017 * Corregido: 25 de noviembre de 2017 * Aceptado: 18 de diciembre de 2017

I. Magister en Administración de Empresas Mención en Negocios Internacionales, Diplomado en Inspección de Soldadura, Ingeniero en Administración Pública, Ingeniero Mecánico, Tecnólogo en Administración Pública, Universidad Técnica Luis Vargas Torres De Esmeraldas, Esmeraldas, Ecuador.

II. Ingeniera en Administración Pública, Tecnólogo en Administración Pública, Docente Instituto Tecnológico Eloy Alfaro, Esmeraldas, Ecuador. 


\title{
Resumen
}

El presente artículo plasma un análisis de los sistemas de información empresariales, donde se describen y se determina, en base a factores como la globalización, la tecnología y la competencia, la importancia de la información actualizada y oportuna para la toma de decisiones, con el fin de crear estrategias que garanticen el correcto funcionamiento de la organización. La creciente globalización, el proceso de internacionalización de la empresa, el incremento de la competencia en los mercados de bienes y servicios, la rapidez en el desarrollo de las tecnologías de información, el aumento de la incertidumbre en el entorno y la reducción de los ciclos de vida de los productos, originan que la información se convierta en un elemento clave para la gestión, así como para la supervivencia y crecimiento de la organización empresarial. La presente se trata de una investigación de carácter documental, en donde se realizó una revisión bibliográfica de varios autores relacionados con el tema en estudio y posteriormente, se realizó un análisis de la información. El estudio descriptivo llevado a cabo, busca especificar los sistemas de información empresarial y su importancia dentro de la toma de decisión gerencial. Ahora bien, se llegó a la conclusión que es imperioso dentro de las empresas, desarrollar estudios que profundicen en los factores a tener en cuenta a la hora de diseñar, implantar y usar los sistemas de información para la dirección en sus funciones de planificación estratégica y de control y supervisión de las tareas operativas y en la definición de los sistemas de procesamiento de datos, de ello depende el éxito o fracaso de la disposición de información adecuada a las necesidades gerenciales y de la empresa, y de la toma de decisiones en pro de la organización.

Palabras clave: sistema; información; empresa; estrategias; decisiones.

\begin{abstract}
This article presents an analysis of business information systems, where they are described and determined, based on factors such as globalization, technology and competence, the importance of updated and timely information for decision making, with the purpose of creating strategies that guarantee the correct functioning of the organization. The increasing globalization, the process of internationalization of the company, the increase of the competition in the markets of goods and services, the rapidity in the development of the information technologies, the increase of the uncertainty in the environment and the reduction of the cycles of life of the products, originate that the information becomes a key element for the management, as well as for the survival and growth of
\end{abstract}


the business organization. This is an investigation of a documentary nature, where a bibliographic review of several authors related to the subject under study was carried out and subsequently, an analysis of the information was made. The descriptive study carried out, seeks to specify the business information systems and their importance in the management decision making. However, it was concluded that it is imperative within companies to develop studies that deepen the factors to be taken into account when designing, implementing and using information systems for management in their strategic planning functions and control and supervision of operational tasks and in the definition of data processing systems, it depends on the success or failure of the provision of information appropriate to the managerial and business needs, and the decision-making process. of the organization.

Keywords: system; information; company; strategies; decisions.

\section{Resumo}

Este artigo apresenta uma análise dos sistemas de informação empresarial, onde são descritos e determinados, com base em fatores como globalização, tecnologia e competência, a importância da informação atualizada e atempada para a tomada de decisões, com a propósito de criar estratégias que garantam o funcionamento correto da organização. A crescente globalização, o processo de internacionalização da empresa, o aumento da concorrência nos mercados de bens e serviços, a rapidez no desenvolvimento das tecnologias da informação, o aumento da incerteza no meio ambiente e a redução dos ciclos. da vida dos produtos, originam que a informação se torna um elemento-chave para a gestão, bem como para a sobrevivência e o crescimento da organização empresarial. Esta é uma investigação de natureza documental, onde foi realizada uma revisão bibliográfica de vários autores relacionados ao assunto em estudo e posteriormente, uma análise das informações foi feita. O estudo descritivo realizado, busca especificar os sistemas de informação comercial e sua importância na tomada de decisões gerenciais. No entanto, concluiu-se que é imperativo que as empresas desenvolvam estudos que aprofundem os fatores a serem levados em consideração ao projetar, implementar e usar sistemas de informação para gerenciamento em suas funções de planejamento estratégico e controle e supervisão de tarefas operacionais e na definição de sistemas de processamento de dados, depende do sucesso ou falha na provisão de informações adequadas às necessidades gerenciais e comerciais e ao processo de tomada de decisão. da organização.

Palavras chave: sistema; informação; empresa; estratégias; decisões. 


\section{Introducción}

La sociedad está basada fundamentalmente en organizaciones y nuestras mayores actividades las realizamos en esa dirección. En la actualidad, las organizaciones se enfrentan a una incertidumbre económica, que requiere de información actualizada y oportuna, con el propósito de lograr sus objetivos y poder satisfacer con sus bienes y servicios a sus clientes y ser el soporte del bienestar del ser humano.

La importancia de la información, es cada día más creciente en la gerencia de las organizaciones, y tiene dentro de sus objetivos básicos, mantenerse y perdurar en el mercado.

Durante los últimos años los sistemas de información, constituyen uno de los principales ámbitos de estudio en el área de organización de empresas. El entorno donde las compañías desarrollan sus actividades, se vuelve cada vez más complejo. La creciente globalización, el proceso de internacionalización de la empresa, el incremento de la competencia en los mercados de bienes y servicios, la rapidez en el desarrollo de las tecnologías de información, el aumento de la incertidumbre en el entorno y la reducción de los ciclos de vida de los productos, originan que la información se convierta en un elemento clave para la gestión, así como para la supervivencia y crecimiento de la organización empresarial. Si los recursos básicos analizados hasta ahora eran tierra, trabajo y capital, ahora la información aparece como otro insumo fundamental a valorar en las empresas.

Según Andreu, Ricart y Valor (1991), un sistema de información queda definido como: “conjunto formal de procesos que, operando sobre una colección de datos estructurada de acuerdo a las necesidades de la empresa, recopila, elabora y distribuyen selectivamente la información necesaria para la operación de dicha empresa y para las actividades de dirección y control correspondientes, apoyando, al menos en parte, los procesos de toma de decisiones necesarios para desempeñar funciones de negocio de la empresa de acuerdo con su estrategia”. Todo sistema de información utiliza como materia prima los datos, los cuales almacena, procesa y transforma para obtener como resultado final información, la cual será suministrada a los diferentes usuarios del sistema, existiendo además un proceso de retroalimentación o "feedback", en la cual se ha de valorar si la información obtenida se adecua a lo esperado. 
Sistemas de información empresarial: la información como recurso estratégico

Junto con los datos, los otros dos componente básicos que constituyen un sistema de información son los usuarios (personal directivo, empleados y en general cualquier agente de la organización empresarial que utilice la información en su puesto de trabajo) y los equipos (informáticos, software, hardware y tecnologías de almacenamiento de la información y de las telecomunicaciones). En muchas ocasiones existe bastante confusión, pues al referirse a sistemas de información se piensa en un primer momento tanto los ordenadores como en los programas informáticos. Una empresa puede adquirir nuevos ordenadores, instalar nuevos productos de telecomunicaciones, elaborar una página web, realizar comercio electrónico, pero ello no implica que exista en su organización un sistema de información. Un sistema de información abarca más que el aspecto meramente computacional, pues no sólo hemos de tener en cuenta estas herramientas, sino también el modo de organizar dichas herramientas y de obtener la información necesaria para el correcto funcionamiento de la empresa.

Ahora bien, Como afirma (Kaye, 1986), todas las organizaciones son sistemas de procesamiento de información. Este enunciado es perfectamente asumido por directivos y responsables de las empresas, ya que cualquier actividad desarrollada en la empresa, necesita procesar información para su funcionamiento. Por su parte, (Andreu, Ricart, \& Valor, 1991) explican como la información se convierte en un recurso estratégico para las empresas y se integra dentro de su proceso de planificación estratégica. Así la información se ha convertido en un recurso clave para las empresas a todos los niveles jerárquicos y para todos los departamentos ya que las organizaciones deben conseguir, procesar, usar y comunicar información, tanto interna como externa, en sus procesos de planificación, dirección y toma de decisiones (Kaye, 1986). Desde un punto de vista económico o empresarial, se ha de resaltar dos hechos clave que confluye en el tiempo. Por un lado, la actual consideración de la información como un recurso crítico estratégico para la gestión empresarial y por otro lado, el amplio desarrollo de las tecnologías de la información, especialmente informática y telecomunicaciones que han dado lugar a importantes transformaciones en la forma de dirigir los negocios. En efecto, hasta la década de los años setenta (Ansoff, 1965), la gestión empresarial se centraba en la adecuada administración de los recursos clásicos de "tierra o energía, trabajo y capital", toda vez que las empresas se encontraban ante un entorno estable y predecible y con una demanda creciente. Bajo estas circunstancias el éxito de las empresas descansaba en la competencia basada en los recursos tangibles, vía costes y en la consecución de economías de escala. En los umbrales del siglo XXI, las empresas se vienen enfrentando a nuevos escenarios, caracterizados por la globalización e internacionalización 
de los negocios, que nos plantean la plena integración en la Unión Europea y los rápidos y continuos cambios que se producen en la economía local, regional, nacional y mundial junto a la heterogénea naturaleza cultural, social, tecnológica, económica, competitiva y de otra índole de las variables que configuran tales cambios. Este nuevo contexto impone a las empresas la necesidad vital de disponer de adecuadas infraestructuras de comunicación y de sistemas de información, que les permitan obtener los datos necesarios a fin de conseguir un conocimiento real y suficiente del entorno que afectará a sus procesos de toma de decisiones y de planificación (Cortés \& García, 1997).

La información como base fundamental para la gerencia según (Cornella, 1994), expresa que las empresas están solicitando información, con mayor regularidad, por la globalización de los mercados, lo cual hace que los gerentes estén más pendientes de lo que sucede en cualquier parte y de lo que hacen sus clientes y competidores.

Para profundizar sobre la temática, (Drucker, 1996), sostiene que la información es la herramienta que hace ver los negocios de una manera diferente, por lo tanto la alta gerencia debe requerir la información en el momento adecuado.

La información necesaria básicamente para la gerencia es: "información básica, información de productividad, información de competencia e información de asignación de recursos, con la finalidad de generar riquezas, y que la empresa continúe en marcha; ésta información permite dirigir la táctica. Para la estrategia la alta gerencia requiere información organizada del entorno".

Asimismo, sostiene (Drucker, 1996), la estrategia debe basarse en información relacionada con los mercados, clientes y no clientes; la tecnología propia de la empresa y de la competencia, finanzas en el ámbito mundial y el ambiente económico mundial.

Por lo tanto, el gerente tiene que estar informado, tanto de sus subordinados como de la red de su entorno, lo cual le permite reunir información de suma importancia, para dirigir la táctica y la estrategia de la organización.

Asimismo, la consecución de una ventaja competitiva utilizando los sistemas de información, dependerá en gran medida del correcto desarrollo y puesta en funcionamiento del sistema de información. El desarrollo de un sistema de información no resulta sencillo. Aquellas organizaciones 
que simplemente adquieren tecnologías de información, sin tener en cuenta las necesidades existentes en la compañía fracasarán, poniendo en peligro la supervivencia de la empresa.

El mismo autor, caracteriza al ejecutivo actual como aquel que administra sin autoridad de mando, lo cual, implica que la alta gerencia debe tener un equipo, que permita dirigir y recibir información oportuna para la toma de decisiones.

De lo mencionado anteriormente, el gerente tiene el papel principal en la toma de decisiones, sólo él puede comunicar, negociar, educar, consultar, emprender y comprometer a la organización en nuevos cursos de acción importantes; y tiene la información suficiente y actualizada para tomar decisiones que determinen la estrategia de la organización.

No obstante, lo amplio que puede ser el panorama de oportunidades de la tecnología de información, para mantener y aumentar la competitividad de las organizaciones, debe tenerse presente el adecuado manejo de la información, como cualquier otro recurso financiero, humano, etc. Por lo tanto, la gestión tecnológica es fundamental como herramienta para planificar el uso de la información en la organización y definir su relación con las áreas funcionales como mercadeo, producción, finanzas y recursos humanos.

Es indispensable para la organización, aprender a identificar los cambios tecnológicos y su relación con las oportunidades del mercado y las amenazas presentes en él, así como la capacidad de diseñar las estrategias para acceder y utilizar adecuadamente la tecnología, para aprovechar las oportunidades o enfrentar las amenazas del ambiente donde compite.

\section{Materiales y métodos}

La presente investigación es de carácter documental, realizándose una revisión bibliográfica y un análisis de la información.

En la abundante literatura existente, sobre la técnica de investigación documental, destacan las siguientes definiciones: (Baena, 1985), "La investigación documental es una técnica que consiste en la selección y recopilación de información, por medio de la lectura y crítica de documentos y materiales bibliográficos, de bibliotecas, hemerotecas, centros de documentación e información, “Según (Garza, 1988), presenta una definición más específica de la investigación documental. Este 
autor considera que ésta técnica “...se caracteriza por el empleo predominante de registros gráficos y sonoros como fuentes de información...", registros en forma de manuscritos e impresos, Se pretende con la misma, en base al estudio de diferentes autores, esbozar, analizar y concluir acerca del tema en cuestión.

Por otra parte, los estudios descriptivos "buscan especificar la propiedades, las características y los aspectos importantes del fenómeno que se somete a análisis. (Gómez, 2006).

Así mismo, de la literatura antes referida, sirve de apoyo para fundamentar éste trabajo investigativo, puesto que en dicha fuente se explica que los estudios descriptivos, se enfocan en recabar datos que muestren cómo se manifiesta un hecho, contexto, evento, fenómeno o situación. De la misma manera, el autor refiere que la descripción puede ser más o menos profunda, y en cualquiera de los casos se basa en la recolección de datos sobre éste y su contexto, en relación específica con las investigaciones de variable cualitativa, como es el presente caso.

\section{Resultados}

Ahora bien, la gestión tecnológica es una actividad esencial en cualquier tipo de organización, ya sea pública o privada. Ésta logra manejar más eficientemente la información, fortalecer los recursos existentes e incrementar las habilidades y destrezas del personal y de la organización de rápido aprendizaje. En virtud de ello, la gestión tecnológica hace de la innovación el elemento o factor disparador más importante, desarrollando una cultura organizacional totalmente identificada con esta forma de conducir cualquier tipo de actividad, con lo cual se facilita la entrada de nuevos bienes y servicios, o de cambios en los procesos de producción o de entrega de los mismos, sin descuidar el recurso de la información, fundamental en la sociedad del conocimiento.

En la era actual, con la creciente globalización y el avance tecnológico, es de vital importancia el manejo de la información actualizada y oportuna con la finalidad de que los gerentes empresariales, programen las estrategias necesarias para el correcto funcionamiento de la empresa, para ello resulta imperioso el mejor desarrollo y uso de los sistemas de información.

El proceso de desarrollo de los sistemas de información, constaría de siete etapas fundamentales: 
1. Definición del proyecto: en esta etapa se determinaría si la empresa presenta problemas y como esto pueden solucionarse mediante la implantación de un sistema de información. En ella se identificarán cuáles son los objetivos del uso de los 9 sistemas de información y como estos se ubican dentro de la estrategia global de la compañía. En esta fase resulta fundamental, que la alta dirección considere los sistemas de información como un arma estratégica y crea realmente en ello.

2. Análisis de sistemas: tras haber identificados los diferentes problemas de la organización, estos serán analizados más detenidamente, identificando las causas que lo originan y planteando diversas soluciones. En esta fase se producirá un estudio de factibilidad, para ver si las soluciones son posibles dados los recursos que posee la organización. Hablaremos de tres tipos de factibilidad:

- Factibilidad técnica: para que la solución pueda ser implantada, hemos de analizar si la empresa posee los medios informáticos adecuados, o bien si hemos de adquirirlos en el exterior.

- Factibilidad económica: se realizara un estudio y valoración económica de la solución, comprobando que los beneficios de la mejora superan los costes de la implantación o modificación de los sistemas de información.

- Factibilidad operativa: hemos de valorar si la solución propuesta es deseable data la organización interna de la empresa.

3. Diseño de Sistemas: una vez elegida aquella solución que resuelva los problemas, se detallará cómo el sistema de información satisface los requisitos planteados por la organización. A la hora de diseñar los sistemas, hemos de indicar que componentes de los sistemas de información utilizaremos (nivel hardware, software y tecnología de las telecomunicaciones) y como se relacionarán dichos componentes entre sí. De esta forma se producirá las especificaciones del sistema de información.

4. Programación: se traducirán las especificaciones del sistema desarrolladas en la etapa anterior, llevándose a cabo la programación y el desarrollo del software.

5. Fase de pruebas: para evaluar el correcto funcionamiento del sistema de información, será necesario llevar a cabo un proceso exhaustivo y profundo para determinar si el sistema de información, funciona en diversas condiciones y si los resultados se corresponden con lo que se esperaba. A la hora de establecer las pruebas, las empresas pueden realizarlas de tres tipos: 
- Pruebas de programas: los diversos programas elaborados se probaran por separado, con el fin de garantizar que cada uno de ellos está libre de errores.

- Pruebas al sistema: se probará el sistema de información como un todo. La finalidad será el correcto funcionamiento del sistema en conjunto, puesto que en ocasiones puede que los programas funcionen correctamente de forma individual, pero a la hora de funcionar en conjunto, el sistema de información no ofrezca los resultados esperados por la empresa.

- Pruebas de aceptación: pruebas realizadas por los usuarios finales del sistema de información. Cuando estos dan el visto bueno se proporciona la certificación final del correcto funcionamiento del sistema de información.

6. Conversión: una vez comprobado que el sistema de información, funciona correctamente se llevará a cabo la implantación de este, o bien la sustitución del antiguo sistema de información por el nuevo. A la hora de llevar a cabo esta conversión, las organizaciones van a poder optar por diversas estrategias:

- La denominada estrategia en paralelo: en ella durante un periodo de tiempo, ambos programas van a convivir y utilizarse, funcionando tanto el nuevo sistema de información como el antiguo. Esta estrategia es la más fiable y segura, aunque sin embargo es la más costosa y podemos obtener información redundante.

- Se puede optar por un cambio directo, remplazando el viejo sistema de información, por el nuevo en una fecha determinado. Esta estrategia sería la menos costosa, sin embargo ante cualquier problema que surja, se puede originar la paralización de la actividad de la empresa. Igualmente requiere que el personal de la compañía, haya recibido formación sobre el nuevo sistema de información, si no nos encontraremos con empleados incapaces de saber manejar el nuevo sistema de información.

- Llevar a cabo una experiencia piloto, donde el nuevo sistema de información se utiliza en un área limitada de la compañía y tras comprobar su correcto funcionamiento se instala en la totalidad de la compañía. Sería una mezcla de las dos estrategias anteriores.

7. Producción y mantenimiento: una vez instalado el nuevo sistema de información, se dice que el sistema está en producción. A partir de aquí, existir un proceso constante de evaluación del sistema 
de información por parte de los usuarios y personal especializado. Tras ello se identificaran nuevos errores y se planteará la corrección de estos.

La totalidad de las fases analizadas, constituirían el denominado ciclo de vida de los sistemas de información. Sin embargo para muchas compañías desarrollar el sistema de información, siguiendo la totalidad de las etapas anteriores puede resultarle muy costoso tanto en tiempo como en dinero. Otros inconvenientes vendrían dados por los continuos cambios de los requisitos de la información, que puede originar que un sistema de información quede obsoleto, incluso en la etapa de desarrollo. Por ello las empresas a la hora de desarrollar un sistema de información, puede optar por otro conjunto de estrategias que le pueden permitir obtener resultados tan positivos como los conseguidos, utilizando el ciclo de vida de los sistemas de información.

El desarrollo e implantación de los sistemas de información, en muchas ocasiones termina en fracaso, lo cual implica un alto coste para la empresa y la pérdida de recursos que se podían haberse utilizado en usos alternativas. A continuación vamos a realizar un análisis a modo de resumen de las principales causas que originan el fracaso de los sistemas de información:

a) Falta de alineación entre los sistemas de información y la estrategia empresarial: muchas organizaciones siguen considerando los sistemas de información como un mero instrumento que simplifica la burocracia sin valorar las ventajas estratégicas que estos presentan.

b) Escaso apoyo de la administración: la alta dirección de la compañía, ha de percibir realmente que los sistemas de información, constituyen un arma estratégica. Además ha de existir una predisposición a cambiar la organización empresarial si lo requieren los nuevos sistemas de información.

c) Mala identificación de las necesidades de información: las empresas implantan las tecnologías de información sin previamente haber realizado un proceso de determinación de las necesidades de información y como estas pueden ser satisfecha utilizando adecuadamente los sistemas de información.

d) Escasa involucración o influencia del usuario final: a la hora de diseñar el sistema de información, resulta fundamental contar con la opinión del usuario final, el cual va a ser quien utilice el sistema de 
información. Por ello este usuario ha de estar motivado e incentivado a colaborar en el diseño del sistema.

e) Nula formación del personal: se requiere siempre la realización de actividades formativas para el aprendizaje de las nuevas herramientas informáticas a utilizar en la empresa.

\section{Conclusiones}

Es determinante en cualquier empresa ya sea pequeña o muy grande, empezar a incluir sistemas que nos faciliten las operaciones diarias y más que eso que nos hagan más productivos a la hora de realizar nuestro trabajo, desde sistemas transaccionales hasta sistemas de información, para ejecutivos que reúnan la información de toda la operación de la empresa y la presenten de manera resumida y digerible esto claro sin perder la objetividad y credibilidad y por qué no sistemas a cualquier nivel de la empresa, que me permitan tomar estrategias adecuadas y medios para lograr el tan anhelado crecimiento empresarial.

En primera instancia, para instalar un sistema de información empresarial, es necesario identificar las principales necesidades y evaluar distintos métodos de satisfacción, teniendo presente en todo momento cuáles son las tecnologías de información disponibles en el mercado y como estas pueden utilizarse. Además han de definirse claramente cuáles son los objetivos de los sistemas de información.

Adecuar los sistemas de información a los recursos de capital y las necesidades de la organización. La posesión de la compañía, los ordenadores más avanzados, los mejores programas y la mejor red de telecomunicaciones no resulta indicativo de un mejor sistema de información, pues en ocasiones puede que con tecnologías de información más modestas se satisfagan de igual manera las necesidades de la compañía. Por ello toda empresa ha de considerar los sistemas de información como un todo, un elemento más de su política de negocio.

Con relación e ello, parece relevante desarrollar estudios que profundicen en los factores a tener en cuenta a la hora de diseñar los sistemas de información, para la dirección en sus funciones de planificación estratégica y de control y supervisión de las tareas operativas y en la definición de los 
sistemas de procesamiento de datos. Así también resultará importante la identificación de la información estratégica y táctica a utilizar por los directivos.

La gestión tecnológica implica la administración efectiva de la información y de los cambios tecnológicos, así como todas las actividades que permiten a la organización hacer el mejor uso de la ciencia y la tecnología, generada interna o externamente. Las organizaciones deben aceptar el desafío de la innovación, deben anticiparse al futuro, a las consecuencias de la innovación, a la reacción de los consumidores, de los competidores y del ambiente de negocios. La innovación debe ser un proceso guiado por la intuición, la inteligencia y la previsión.

Ahora bien, resulta imprescindible disponer de la habilidad técnica y económica necesaria para diseñar y desarrollar un sistema de información, que conecte de forma permanente a la empresa con las fuentes de información externas. En tal sentido parece conveniente la realización de estudios que traten de determinar las fuentes de información externas más precisas para cada tipo de empresa y sector industrial.

\section{Bibliografía}

Andreu, R., Ricart, J., \& Valor, J. (1991). Estrategia y sistemas de información. Madrid: Mc Graw Hill

Ansoff, H. (1965). Corporate Strategy. New York: McGraw-Hill

Baena, G. (1985). Manual para elaborar trabajos de investigación documental. México: Mexicanos Unidos

Cornella. (1994). Los recursos de información. Ventaja competitiva de las empresas. Madrid: McGraw-Hill Interamericana España, S.A

Cortés, \& García. (1997). Reflexiones en torno a la dimensión estratégica de las tecnologías de la información en la empresa. Esic-Market (95), 63-77

Drucker, P. (1996). Su visión sobre: la administración, la organización, la economía, la sociedad. Bogotá: Norma 
Sistemas de información empresarial: la información como recurso estratégico

Garza, E. d. (1988). Garza Toledo, Enrique, hacia una metodología de la reconstrucción: fundamentos, crítica y alternativas a la metodología y técnicas de investigación social. México: Porrúa

Gómez, M. (2006). Introducción a la metodología de la investigación científica. (1ra.). Córdoba, Argentina: Brujas

Kaye, D. (1986). An information model of organization. Managing information, 19-21 